\title{
Evaluation of the new Id-Fungi plates from Conidia for MALDI-TOF MS identification of filamentous fungi and comparison with conventional methods as identification tool for dermatophytes from nails, hair and skin samples
}

\author{
Rosalie Sacheli $^{1}$ (D) | Anne-Sophie Henri ${ }^{1}$ | Laurence Seidel $^{2}$ | Marie Ernst $^{2}$ | \\ Rajae Darfouf $^{1}$ | Caroline Adjetey ${ }^{1}$ | Marjorie Schyns ${ }^{1}$ | Ludwig Marechal ${ }^{3}$ | \\ Cécile Meex $^{1}$ | Jorge Arrese ${ }^{3}$ | Marie-Pierre Hayette ${ }^{1}$
}

\footnotetext{
${ }^{1}$ Department of Clinical Microbiology, National Reference Center for Mycosis, Center for Interdisciplinary research on Medicines (CIRM), University Hospital of Liege, Liege, Belgium

${ }^{2}$ Department of Biostatistics, University Hospital of Liege, Liege, Belgium

${ }^{3}$ Department of Dermatopathology, University Hospital of Liege, Liege, Belgium

\section{Correspondence}

Sacheli Rosalie, Department of Clinical Microbiology, National Reference Center for Mycosis, Center for Interdisciplinary research on Medicines (CIRM), University Hospital of Liege, Liege, Belgium.

Email: r.sacheli@chuliege.be
}

\begin{abstract}
Objectives: We first compare the efficiency of mould/dermatophyte identification by MALDI-TOF MS using a new medium called Id-Fungi plates (IDFP) from Conidia ${ }^{\circledR}$ and two different databases. For the second purpose, we evaluated a new version of the medium supplemented with cycloheximide, Id-Fungi plates Plus (IDFPC) for the direct inoculation of nails, hair and skin samples and compared the efficiency of MALDI-TOF MS identification of dermatophytes to classical methods based on culture and microscopy.

Methods: A total of 71 strains have been cultured IDFP and Sabouraud gentamicin plates (SGC2) and were identified by MALDI-TOF MS. For the evaluation of the combination IDFPC/ MALDI-TOF MS as a method of identification for dermatophytes, 428 samples of hair nails and skin were cultivated in parallel on IDFPC and Sabouraud + cycloheximide medium (SAB-ACTI).

Results: For Aspergillus sp and non-Aspergillus moulds, the best performances were obtained on IDFP after maximum 48-h growth, following protein extraction. For dermatophytes, the best condition was using the IDFP at 72 hours, after extended direct deposit. Regarding the direct inoculation of nails, hair skin on IDFPC, 129/428 (30.1\%) showed a positive culture against 150/428 (35\%) on SAB-ACTI medium. Among the 129 positive strains, the identification by MALDI-TOF MS was correct for $92 / 129$ (71.4\%).

Conclusion: The IDFP allows the generation of better spectra by MALDI-TOF MS compared to SGC2. It facilitates sampling and deposit. Regarding the use of IDFPC, this medium seems less sensitive than SAB-ACTI but among positive strains, the rate of correct identification by MALDI-TOF MS is satisfactory.

KEYWORDS

dermatophytes, Id-Fungi plates, MALDI-TOF MS
\end{abstract}




\section{1 | INTRODUCTION}

Matrix-assisted laser desorption/ionisation-time-of-flight mass spectrometry or MALDI-TOF MS has become the 'gold standard' for routine identification of most microbial organisms in clinical laboratories and has largely replaced conventional identification methods such as biochemical assays. A huge success has been obtained with this method regarding bacteria and yeasts, leading to fast, inexpensive and accurate identification. ${ }^{1-5}$ Regarding filamentous fungi and particularly dermatophytes, a lot of studies have shown a variable success rate for the correct identification by MALDI-TOF MS. ${ }^{6,7}$ This method can be technically difficult to implement routinely for non-yeast fungal species leading to the maintenance of conventional methods for identification. Nevertheless, these methods are fastidious, time-consuming and require huge technician expertise. Applying a new diagnostic approach using the MALDI-TOF mass spectrometry-based strategy should facilitate the effective identification of clinical fungal isolates. Moreover, this approach should lead to a drastic reduction in the response times in routine clinical laboratories what is an essential point when we know that conventional methods based on culture and microscopy can lead to a turnaround time reaching sometimes 3 weeks. One of the major challenge of MALDI-TOF MS identification of filamentous fungi is the sampling of the fungal culture and deposit on the target as the culture is sometimes hardly adhering to the culture media. To facilitate the growth and sampling of filamentous fungi, the new medium Id-Fungi plates ${ }^{\circledR}$ (IDFP) from Conidia Laboratory (France) has been evaluated on strains in a first time and then we evaluated an improved version of this medium containing cycloheximide (IDFPC) as a tool of identification directly on nails, hair and skin samples. This medium has the particularity to be covered by a porous membrane allowing nutriment and carbon exchanges but limiting cellular adhesion. We thus in a first approach compared these plates to the classical Sabouraud dextrose medium (SGC2), evaluating the ability of this new medium to give rise to correct identification of Aspergillus sp, non-Aspergillus sp moulds and dermatophytes by MALDI-TOF MS. Then, we evaluated the combination of an improved version of IDFPC with MALDITOF MS as a tool for the identification directly on nails, hair and skin samples. The medium was here compared to Sabouraud + cycloheximide (SAB-ACTI) medium. One next challenge for the identification of filamentous fungi by MALDI-TOF MS is the database used. Indeed in the past, few databases were developed for filamentous fungi and commercially available databases were too incomplete to obtain good rates of identifications particularly for dermatophytes. The only encouraging results obtained were done with 'In house' databases implemented by laboratory users. ${ }^{8,9}$ Fortunately, efforts have been done worldwide to make databases available for public access to permit clinical microbiology laboratories to more broadly use MALDI-TOF MS for moulds identification. ${ }^{10,11}$ A new library, the 'Mass Spectrometry Identification' (MSI) library, has recently been published by Normand et al and currently contains 938 species belonging to 246 genera (11 815 spectra for 1913 strains). ${ }^{11}$ This database has been evaluated in our work on strains in culture, and results were compared to those obtained with Bruker 2.0 filamentous fungi database, a commercially available application that covers 152 species/species groups and 468 spectra, for which contributions have been received from over 20 laboratories across 8 countries.

\section{2 | MATERIAL AND METHODS}

\section{1 | Isolates and culture conditions}

\subsubsection{Comparison IDFP vs SGC2}

For the first part of the evaluation (comparing IDFP vs SGC2 for MALDI-TOF MS identification of fungi), a total of 71 strains were tested as follows: 13 Aspergillus species, 26 non-Aspergillus moulds and 32 dermatophytes (see Tables 1 and 2 for species description). These strains were provided by the NRCM collection (University Hospital of Liège, Belgium) or by the Belgian Coordinated Collections of Microorganism-Scientific Institute of Public Health (BCCM/IHEM; Brussels, Belgium). The isolates were frozen and subcultured twice prior to inoculation on IDFP and SGC2 medium. Frozen cultures were grown first on SGC2 at $30^{\circ} \mathrm{C}$ and were incubated between 2 and

TABLE 1 Number of correct identifications obtained for the different Aspergillus and non-Aspergillus moulds tested in this study, considering the Bruker 2.0 filamentous fungi database with a complete protein extraction method

\begin{tabular}{|c|c|c|c|c|}
\hline \multirow[b]{2}{*}{ Fungal species } & \multicolumn{2}{|l|}{ IDFP } & \multicolumn{2}{|c|}{ SGC2 } \\
\hline & $24 \mathrm{~h}$ & $48 \mathrm{~h}$ & $24 \mathrm{~h}$ & $48 \mathrm{~h}$ \\
\hline Aspergillus fumigatus & $3 / 5$ & $5 / 5$ & $1 / 5$ & $1 / 5$ \\
\hline Aspergillus flavus & $2 / 2$ & $2 / 2$ & $1 / 2$ & $1 / 2$ \\
\hline Aspergillus nidulans & $2 / 2$ & $2 / 2$ & $1 / 2$ & $0 / 2$ \\
\hline Aspergillus terreus & $1 / 1$ & $1 / 1$ & $1 / 1$ & $0 / 1$ \\
\hline Aspergillus niger & $3 / 3$ & $2 / 3$ & $3 / 3$ & $2 / 3$ \\
\hline Lomentospora prolificans & $1 / 1$ & $1 / 1$ & $1 / 1$ & $1 / 1$ \\
\hline Scedosporium apiospermum & $0 / 1$ & $1 / 1$ & $1 / 1$ & $1 / 1$ \\
\hline Fusarium dimerum & $1 / 1$ & $1 / 1$ & $1 / 1$ & $1 / 1$ \\
\hline Fusarium oxysporum & $3 / 3$ & $3 / 3$ & $3 / 3$ & $3 / 3$ \\
\hline Paecymolyces lilacinus & $1 / 2$ & $2 / 2$ & $1 / 2$ & $2 / 2$ \\
\hline Lichteimia corymbifera & $2 / 2$ & $2 / 2$ & $0 / 2$ & $1 / 2$ \\
\hline Rhizomucor pusilus & $1 / 1$ & $1 / 1$ & $0 / 1$ & $1 / 1$ \\
\hline Mucor velutinosus & $0 / 1$ & $0 / 1$ & $0 / 1$ & $0 / 1$ \\
\hline Curvularia lunata & $1 / 1$ & $1 / 1$ & $0 / 1$ & $1 / 1$ \\
\hline Rasamsonia argillacea & $1 / 3$ & $2 / 3$ & $1 / 3$ & $2 / 3$ \\
\hline Acremonium sclerotigenum & $0 / 1$ & $0 / 1$ & $0 / 1$ & $0 / 1$ \\
\hline Alternaria alternata & $3 / 3$ & $3 / 3$ & $3 / 3$ & $1 / 3$ \\
\hline Scopulariopsis brevicaulis & $4 / 4$ & $0 / 4$ & $3 / 4$ & $4 / 4$ \\
\hline Neoscitalidium dimidiatum & $0 / 1$ & $0 / 1$ & $0 / 1$ & $0 / 1$ \\
\hline Penicillium chrysogenum & $1 / 1$ & $0 / 1$ & $1 / 1$ & $1 / 1$ \\
\hline
\end{tabular}

Note: Abbreviations: IDFP, ID-Fungi plates medium; SGC2, Sabouraud gentamicin-chloramphenicol medium. 
TABLE 2 Number of correct identifications obtained for the different dermatophytes tested in this study, considering the Bruker 2.0 filamentous fungi database with an extended direct deposit method

\begin{tabular}{|c|c|c|c|c|c|c|}
\hline \multirow[b]{2}{*}{ Fungal species } & \multicolumn{3}{|l|}{ IDFP } & \multicolumn{3}{|c|}{ SGC2 } \\
\hline & $48 \mathrm{~h}$ & $72 \mathrm{~h}$ & $96 \mathrm{~h}$ & $48 \mathrm{~h}$ & $72 \mathrm{~h}$ & $96 \mathrm{~h}$ \\
\hline Trichophyton interdigitale & $7 / 8$ & $6 / 8$ & $1 / 8$ & $3 / 8$ & $1 / 8$ & $3 / 8$ \\
\hline Trichophyton benhamiae & $0 / 5$ & $5 / 5$ & $2 / 5$ & $0 / 5$ & $2 / 5$ & $3 / 5$ \\
\hline Trichophyton rubrum & $3 / 8$ & $6 / 8$ & $3 / 8$ & $4 / 8$ & $7 / 8$ & $8 / 8$ \\
\hline Trichophyton tonsurans & $0 / 5$ & $4 / 5$ & $0 / 5$ & $0 / 5$ & $5 / 5$ & $1 / 5$ \\
\hline Trichophyton violaceum & $0 / 2$ & $0 / 2$ & $0 / 2$ & $0 / 2$ & $0 / 2$ & $0 / 2$ \\
\hline Microsporum canis & $1 / 4$ & $4 / 4$ & $2 / 4$ & $2 / 4$ & $4 / 4$ & $1 / 4$ \\
\hline
\end{tabular}

Note: Abbreviation: SGC2, Sabouraud gentamicin-chloramphenicol medium; IDFP, ID-Fungi plates medium.
21 days. When growth was observed, the inoculation on Id-Fungi plates (IDFP, Conidia, France, https://conidia.fr) and Sabouraud gentamicin-chloramphenicol (SGC2, bioMérieux, France) was done. Incubation at $30^{\circ} \mathrm{C}$ was performed between 24 hours and 96 hours, depending on the fungal species.

\subsection{2 | Comparison of IDFPC + MALDI-TOF MS vs the conventional method for dermatophytes identification}

For the second part of the evaluation concerning the comparison of the combination IDFPC + MALDI-TOF MS with the conventional method consisting of a culture on SAB-ACTI followed by microscopic identification, 428 samples of nails, hair and skin were treated including 258 nails, 10 hairs and 160 skin samples. A direct examination was done for hair, skin and nails samples, microscopic examination by histology with PAS staining was applied on every sample. These samples were then inoculated in parallel on IDFPC and Sabouraud dextrose agar in tubes with chloramphenicol and cycloheximide (SAB CHL ACTI-T, bioMérieux, France). IDFPC showed a modified composition compared to the one (IDFP) tested for the comparison with SGC2; indeed, cycloheximide was not present on the first medium composition and was added to improve dermatophyte culture and avoid contamination. Incubation was performed at $30^{\circ} \mathrm{C}$ until visible growth. Samples were classified into three categories, 'dermatophytes', 'contaminants' concerning samples with culture positive other than dermatophytes and direct examination negative and 'Potentially pathogenic Mould' concerning samples with positive culture other than dermatophytes and a positive direct examination.

\section{2 | DNA sequencing}

All 71 strains that were used for the comparison IDFP vs SGC2 plates for MALDI-TOF MS identification and all 129 strains that were positive in culture after nails, skin or hair inoculation on IDFPC were identified by DNA sequencing as a reference method for species confirmation. The rRNA ITS2 region was sequenced for all strains.
Briefly, DNA was extracted from a mycelial mass (cultured in liquid Sabouraud dextrose broth) using the Maxwell SEV 16 cell kit (Promega) with the following pretreatment: Briefly, after growth, the liquid culture was centrifuged at $3000 \mathrm{~g}$ for 10 minutes; the pellet culture was immersed into $1 \mathrm{~mL}$ of water placed onto tubes with $0.5 \mathrm{~mm}$ microbeads (Omni international). Samples were vortexed for 30 minutes. Then, the liquid was removed and a chemical lysis was done with $20 \mu \mathrm{L}$ Proteinase $\mathrm{K}$ on $200 \mu \mathrm{L}$ ATL buffer and $180 \mu \mathrm{L}$ AL buffer (all three manufactured by Qiagen). Following this described pretreatment, DNA was extracted with the Maxwell ${ }^{\circledR}$ (Promega) instrument following the manufacturer's instructions. The ITS2 region was amplified using the ITS86 forward primer $5^{\prime}$ GTG-AAT-CAT-CGAATC-TTT-GAA $3^{\prime}$ and ITS4 reverse primer 5'TCC-TCC-GCT-TATTGA-TAT-GC $3^{\prime} .^{12}$ Purification of PCR products was then performed using the Exosap IT technique (Amersham, GE Healthcare Europe $\mathrm{GmbH}$, Belgium). PCR was done on a classical thermocycler (Thermo Hybaid, Thermo Scientific). Bidirectional sequence data were generated after purification using the BigDye terminator sequencing kit (Applied Biosystems, Life technologies, Belgium). Sequenced products were finally purified using the kit clean Seq Agencourt (Beckman Coulter Life Science). The sequencing was done on the automate ABI 3500/3500XL (Applied Biosystem, Life Technologies). Sequences were edited using the ABI Sequence Scanner V.1.0 software (Applied Biosystems, Life Technologies). Sequences generated by the software were then compared to the CBS database, which comprises several databases including Genbank.

\section{3 | DermaGenius ${ }^{\circledR}$ real-time multiplex PCR}

If no identification was obtained by ITS sequencing as it was the case for 11 strains, a DermaGenius ${ }^{\circledR}$ real-time multiplex PCR (PathoNostics) was applied. Extraction was performed on strains in culture. A piece of culture was scrapped from the IDFPC and immerged into $475 \mu \mathrm{L}$ of ATL buffer (Qiagen). $25 \mu \mathrm{L}$ of proteinase $\mathrm{K}$ was added to each tube. An incubation at $56^{\circ} \mathrm{C}$ overnight was done under agitation at $900 \mathrm{rpm}$. After this pretreatment, a DNA extraction was done using the QIAamp DNA minikit (Qiagen) following the manufacturer's instructions. The PCR was also performed according to manufacturer's instructions: $5 \mu \mathrm{L}$ of extracted DNA was added 
to the PCR mix, and a LightCycler 480 II (Roche) was used for amplification and melting curve analysis. Data analysis was done using the 2nd-derivative and Tm-calling function of the LC480 software (version 1.5.1.62 SP2).

\subsection{MALDI-TOF MS sample preparation/ extraction}

For the comparison between IDFP and SGC2, an extended direct deposit was performed by adding $1 \mu \mathrm{L}$ of $70 \%$ formic acid to the sample on a MALDI target plate (MSP 96 BC ground steel target; Bruker Daltonics). Then, $1 \mu \mathrm{L}$ of saturated cyano-4-hydroxycinnamic acid solution (HCCA matrix; Bruker Daltonics) was added. In parallel, formic acid-ethanol protein extraction was performed according to the recommendations of Bruker Daltonics $\mathrm{GmbH}$ (Germany). Briefly, $300 \mu \mathrm{L}$ deionised water was putted to a tube, and the culture was added on the tube from solid culture plates, scrapping with a tip or a scalpel if the culture was hard to pick up. The sample was vortexmixed. $900 \mu \mathrm{L}$ of $100 \%$ ethanol was added to the sample. After the centrifugation of the mixture at maximum speed (15700g) for 2 minutes, the supernatant was discarded, and ethanol was completely removed by drying the pellet 2-3 minutes at ambient temperature. $50 \mu \mathrm{L}$ of $70 \%$ formic acid was then added, and the sample was thoroughly vortex-mixed. Next, an equal volume of acetonitrile was added, and the sample was centrifuged again at $15700 \mathrm{~g}$ for $2 \mathrm{~min}-$ utes. $1 \mu \mathrm{L}$ of the supernatant was finally transferred to a MALD target plate (MSP 96 BC ground steel target; Bruker Daltonics) and covered by $1 \mu \mathrm{L}$ of saturated cyano-4-hydroxycinnamic acid solution (HCCA matrix; Bruker Daltonics). For the second part of the study concerning the evaluation of the combination of IDFPC + MALDITOF MS vs culture on SAB-ACTI and microscopy, an extended direct deposit has been done only as described above. Four spots have been done for the same isolate the result was validated if almost 2 spots gave the same identification and with a score $>1.7$.

\section{5 | MALDI-TOF MS acquisition and databases}

Mass spectra acquisition was done using a Microflex Flex Control V3.4 system (Bruker Daltonics). A Bruker Bacterial Test Standard (BTS255343; Bruker Daltonics) was used for instrument calibration. The acquisition was done at 24 hours postinoculation (on Id-Fungi plates and SGC2 media) and 48 hours for Aspergillus and non-Aspergillus moulds. It was performed at 48 hours, 72 hours and 96 hours postinoculation for dermatophytes. Each microorganism tested was spotted twice on the same target slide. Measurement was performed with MALDI Flex control V3.4 (Bruker Daltonics) following the settings suggested by the manufacturer using automated collecting spectra. The spectra of each duplicated/quadruplicated spot were compared with those in the reference library (Bruker filamentous fungi 2.0) and analysed with MALDI Biotyper software (Bruker Daltonics), with log scores ranging from 0 (no similarity) to 3 (perfect match). The mass spectra-based identification was considered reliable only if at least one out of the two spots resulted in log scores of $\geq 1.70$. No clear recommendations were formulated for filamentous fungi by the manufacturer. Acquired spectra were then submitted to a web-based application designed for microbial mass spectrum identification (MSI application). For identification, the raw mass spectra of a sample were compressed into a zip file and submitted to the web application. Threshold values below 20 were not considered as defined previously by Normand et a ${ }^{13}$ Correct identification at the species level has always been considered in the percentages cited. For the second part of the study concerning the evaluation of the combination of IDFPC + MALDI-TOF MS vs culture on SAB$\mathrm{ACTI}$ and microscopy for the identification of dermatophytes, all the MALDI-TOF MS analysis has been done with Bruker 2.0 Filamentous fungi database and not with MSI database.

\subsection{Statistical analysis}

For statistical analysis, the software SAS has been used (version 9.4). To compare the rate of correct identification obtained by MALDITOF MS between different conditions (different times, IDFP vs SGC2, Bruker vs MSI, extended direct deposit vs extraction), the McNemar test has been used. For the second part of the study concerning the evaluation of the combination of IDFPC + MALDITOF MS vs culture on SAB-ACTI and microscopy for the identification of dermatophytes, the chi-square test or Fisher exact test has been used to compare the proportions between distinct groups, and the McNemar test was used to compare the proportions inside the same group. The results were considered as significant if the $P$-value was $<.05$.

\section{7 | Ethical statement}

The authors confirm they have adhered to Mycoses' ethical policy.

\section{3 | RESULTS}

\subsection{Comparison of the IDFP medium vs SGC2 with Bruker 2.0 filamentous fungi database}

In this work, Aspergillus sp, non-Aspergillus moulds and dermatophyte identification rates obtained by MALDI-TOF MS have been compared between IDFP medium and SGC2. The first observation when using both mediums in parallel was that sampling was clearly easier when using IDFP medium. Indeed, the colony was less adherent to the membrane even for species such as dermatophytes. There was no risk to harvest agar which can alter MALDI-TOF MS identification. Regarding Aspergillus sp, 13 strains of five different species have been tested (see Table 1 for species description). The extended direct deposit method has been compared to formic acid-ethanol 


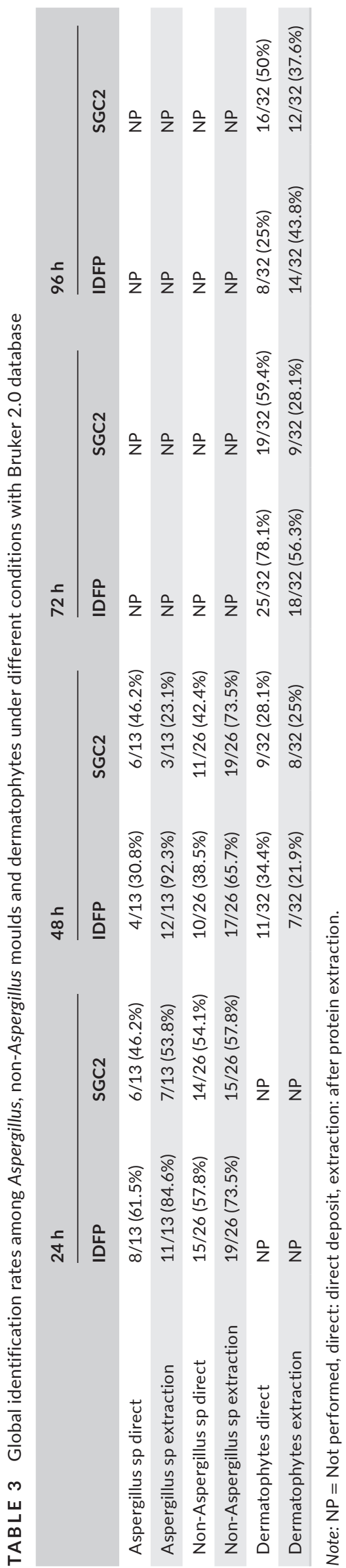

protein extraction after $24-\mathrm{h}$ and 48 -h incubation (Table 3 ). The best condition to obtain $92.3 \%$ of correct identification (12/13) was by using the IDFP medium at 48 hours with formic acid-ethanol protein extraction and using Bruker 2.0 filamentous fungi database. All A fumigatus/A terreus and A flavus strains have been correctly identified at the species level with a good score higher than 1.8. Only one strain of $A$ niger has not been identified under these conditions. The rate of correct identifications obtained with the IDFP medium is significantly higher than this obtained with SGC2 under the same conditions $(23.1 \%, 3 / 13, P$-value $=.006)$. The use of formic acidethanol protein extraction significantly improves the rate of correct identification compared to the extended direct deposit method $(P$-value $=.01)$

Regarding non-Aspergillus moulds, 26 strains from 13 different genera have been tested. This included Lomentospora prolificans, Fusarium dimerum/oxysporum, Penicillium chrysogenum, Acremonium sclerotigenum, Rasamsonia argillaceae, Scytalidium dimidiatum, Alternaria alternata, Scopulariopsis brevicaulis, Lichteimia corymbifera, Mucor velutinosus, Rhizomucor pusillus, Curvularia lunata and Paecilomyces lilacinus. Correct identification at the species level has always been considered in the percentages cited. The results obtained for these genera under different conditions are presented in Table 3. The optimal conditions permitting to reach $73.5 \%(19 / 26)$ of correct identification at the species level were obtained by using the IDFP medium at 24 hours, following formic acid-ethanol protein extraction. The rate of correct identifications obtained with SGC2 is lower than the one obtained with IDFP medium (15/26, 57.8\%). The identification on IDFP at 24 hours of incubation was slightly better than at 48 hours with IDFP medium, but this difference was not statistically significant (17/26, 65.7\%). However, we have to notice that for non-Aspergillus moulds, the extraction on SGC2 medium at 48 hours also permits to reach the rate of $73.5 \%$ of correct ID (19/26). Table 1 described the different species tested and their number of correct identification. Aspergillus and non-Aspergillus species have been considered only for the condition with complete extraction, at 24 hours and 48 hours, these conditions giving the best rates of correct identification.

Regarding dermatophytes, results obtained following different conditions are presented in Table 3 . The best condition to obtain $78.1 \%(25 / 32)$ of correct identification at the species level was with IDFP medium and extended direct deposit, after 72 hours of incubation. The rate of correct identification using the SGC2 medium under the same conditions was lower $(59.4 \%$ of correct identification, 19/32, $P$-value $=.08)$. The rate of correct identification at 72 hours is significantly better than at 48 hours ( $P$ value $=.001)$. Extended direct deposit is better than extraction for dermatophyte identification ( $P$-value $=.05)$. Table 2 shows the number of correct identification by different species of dermatophytes using the Bruker filamentous fungi 2. 0 database and the extended direct deposit method. This method shows the best rates of correct identifications compared to the complete protein extraction method. In tables 4 and 5 are summarised the scores obtained, respectively, with Bruker filamentous fungi 2.0 and MSI 
TABLE 4 Description of the scores obtained with the IDFP medium using Bruker database under optimal conditions for dermatophyte identification (at $72 \mathrm{~h}$, by extended direct deposit)

\begin{tabular}{llllll} 
Fungal Species & $\begin{array}{l}\text { Scores }>2 \text { with } \\
\text { correct ID }\end{array}$ & $\begin{array}{l}\text { Scores }>2 \text { with } \\
\text { incorrect ID }\end{array}$ & $\begin{array}{l}\text { Scores }>1.7 \text { with } \\
\text { correct ID }\end{array}$ & $\begin{array}{l}\text { Scores }>1.7 \text { with } \\
\text { incorrect ID }\end{array}$ & $\begin{array}{l}\text { No } \\
\text { peak }\end{array}$ \\
\hline Trichophyton interdigitale & $5 / 8$ & $1 / 8$ & $1 / 8$ & $0 / 8$ & $0 / 8$ \\
\hline Trichophyton benhamiae & $0 / 5$ & $0 / 5$ & $5 / 5$ & $0 / 5$ & $0 / 5$ \\
\hline Trichophyton rubrum & $3 / 8$ & $0 / 8$ & $3 / 8$ & $0 / 8$ & $0 / 8$ \\
\hline Trichophyton tonsurans & $4 / 5$ & $0 / 5$ & $0 / 5$ & $0 / 5$ & $0 / 5$ \\
\hline Trichophyton violaceum & $0 / 2$ & $0 / 2$ & $0 / 2$ & $0 / 2$ & $0 / 2$ \\
\hline Microsporum canis & $4 / 4$ & $0 / 4$ & $0 / 4$ & $0 / 4$ & $0 / 4$ \\
\hline
\end{tabular}

Note: ID = identification.

\begin{tabular}{|lllll|}
\hline Fungal species & $\begin{array}{l}\text { Scores }>\mathbf{2 0} \text { with } \\
\text { correct ID }\end{array}$ & $\begin{array}{l}\text { Scores }>\mathbf{2 0} \text { with } \\
\text { incorrect ID }\end{array}$ & Scores $<20$ & $\begin{array}{l}\text { No } \\
\text { peak }\end{array}$ \\
\hline Trichophyton interdigitale & $2 / 8$ & $5 / 8$ & $0 / 8$ & $1 / 8$ \\
\hline Trichophyton benhamiae & $4 / 5$ & $0 / 5$ & $0 / 5$ & $1 / 5$ \\
\hline Trichophyton rubrum & $7 / 8$ & $0 / 8$ & $0 / 8$ & $1 / 8$ \\
\hline Trichophyton tonsurans & $1 / 5$ & $3 / 5$ & $0 / 5$ & $1 / 5$ \\
\hline Trichophyton violaceum & $0 / 2$ & $1 / 2$ & $0 / 2$ & $1 / 2$ \\
\hline Microsporum canis & $4 / 4$ & $0 / 4$ & $0 / 4$ & $0 / 4$ \\
\hline
\end{tabular}

TABLE 5 Description of the scores obtained with the IDFP medium using MSI database under optimal conditions for dermatophyte identification (at $72 \mathrm{~h}$ by extended direct deposit)

Note: ID = identification

database. Only the optimal conditions for the best identification of dermatophytes were considered here (at 72 hours with IDFP using extended direct deposit). We can see that with Bruker filamentous fungi 2.0 database, one cross-reaction is observed for $T$ interdigitale. Indeed, a score $>1.7$ is given for this species with the identification $T$ tonsurans. With MSI database, the cross-reactions are more numerous and concern $T$ interdigitale (score higher than 20 with another identification than $T$ interdigitale for $5 / 8$ strains), $T$ tonsurans (score higher than 20 with another identification than $T$ tonsurans in 3/5 strains) and $T$ violaceum (score higher than 20 with another identification than $T$ violaceum in 1/2 strains).

\section{2 | Comparison Bruker filamentous fungi 2.0 VS MSI databases}

The next step was to compare the results obtained using IDFP medium with the commercially available Bruker 2.0 filamentous fungi database and with the MSI application published by Normand et $\mathrm{al}^{14}$ The comparison of both databases is illustrated in Table 6. Regarding Aspergillus sp, a rate of $92.3 \%$ of correct identification (ID) is obtained at 24 hours with a complete protein extraction with MSI while a rate of $84,6 \%(11 / 13)$ is obtained under the same conditions with Bruker database ( $P$-value $=.56)$. However, at 48 hours after extraction, while this condition permits to obtain $92,3 \%$ of correct ID with Bruker 2.0 filamentous fungi database, we only obtain 8/13 (61.5\%) of correct ID under the same conditions using MSI application ( $P$ value $=.046$ ). The results obtained by direct deposit are quite similar using both databases, and the use of MSI database does not permit to increase significantly the rate of correct identifications obtained under these conditions.

Considering non-Aspergillus moulds, using MSI application permit to increase the rate of correct ID obtained after extraction at 48 hours. Indeed, while only $17 / 26$ (65.7\%) strains are correctly recognised by MALDI-TOF MS with the Bruker 2.0 filamentous fungi database, 24/26 (92.5\%) can be efficiently recognised using the MSI application ( $P$-value $=.03$ ). By direct deposit, the identification using MSI application and Bruker 2.0 filamentous fungi database is quite similar, and no statistically significant difference has been shown using one or the other condition on non-Aspergillus moulds.

Regarding dermatophytes, at 48 hours, the results obtained with MSI database are better than with Bruker database (16/32 vs 11/32, $P$-value $=.02)$. At 72 hours, the use of MSI application is not linked with a significant increase on the rates of correct identification. By extended direct deposit, while 78.1\% (25/32) strains are correctly identified with Bruker 2.0 filamentous fungi, $71.9 \%$ (23/32) of the strains are correctly identified with the MSI 2.0 database, so no significant difference is observed under these conditions. No significant difference on the rates of correct identification obtained at 96 hours was observed between both used databases.

\subsection{Evaluation of the combination of IDFPC + MALDI-TOF MS vs culture on SAB-ACTI and microscopy for the identification of dermatophytes}

Nails, hair and skin were inoculated in parallel on IDFPC and SAB-ACTI medium. We thus compared the performance of both 


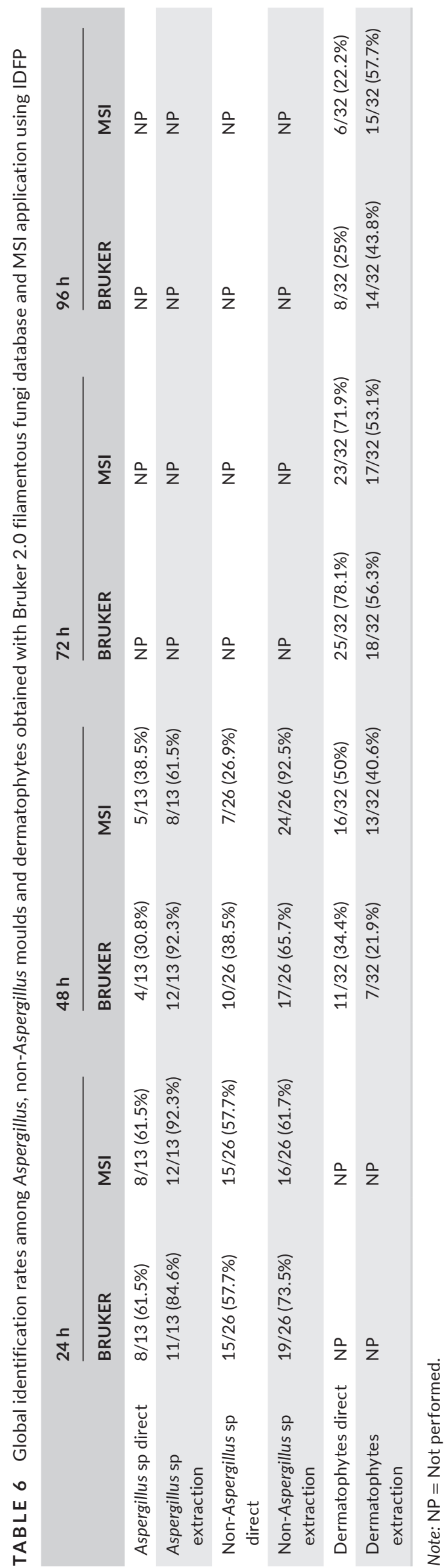

mediums for the growth of dermatophytes. We first observed that IDFPC dry much faster than slants agar tubes along our observation, Moreover, some nails are not totally adhering on IDFPC, giving rise to loss of material. The mean time of positivity on IDFPC was 6 days. The sporulation was observed in parallel on IDFPC and on SAB-ACTI medium. Mycelial elements were in general more abundant on SAB-ACTI medium than on IDFPC. Results of the evaluation are presented in Table 7. Among the 428 samples, 129 (30.1\%) were positive on IDFPC when 150 were positive on SAB-ACTI medium $(35 \%)(P$-value $=.02)$. Considering the IDFPC, 91(70.1\%) samples were dermatophytes, 25 (19.4\%) were contaminants and 13 (10.1\%) were potentially pathogenic moulds. Regarding SAB-ACTI medium, 115 were dermatophytes (76.7\%), 30 (20.1\%) were contaminants, $4(2.7 \%)$ were potentially pathogenic moulds, and $1(0.7 \%)$ tube showed a mixed population of dermatophyte and contaminants. So more dermatophytes are growing on SAB-ACTI ( $P$-value $=.0002)$ compared to IDFPC. However, a little more potentially pathogenic fungi are revealed with IDFPC compared to SAB-ACTI medium $(P$-value $=.02)$. Table 8 shows that among samples that present a negative direct examination, 39 (14\%) had a positive culture on IDFPC and $53(19 \%)$ had a positive culture on SAB-ACTI medium $(P$-value $=.048)$. Among these positive cases with a negative direct examination, the proportion of dermatophytes is higher for the SABACTI medium (25/279, 9\%) than for the IDFPC medium (14/279, 5\%) $(P$-value $=.002)$. Among samples with a positive direct examination, 90 (60.4\%) were positive in culture on IDFPC and 97 (65.1\%) were positive on SAB-ACTI but this difference is not statistically significant $(P$-value $=.25)$. However among the positive samples, the proportion of dermatophytes is higher on SAB-ACTI medium $(91 / 149,61.1 \%)$ than on IDFPC (77/149, 51.7\%) ( $P$-value $=.01)$. No statistically significant difference is observed regarding contaminants. However among the positive direct examination samples, the proportion of non dermatophytes moulds potentially pathogenic is higher on IDFPC than on SAB-ACTI ( $P$-value $=.02$ ).

Table 9 described the repartition of samples on both studied mediums regarding the origin of the sample (nails, hair or skin scrapings). We can observe that among nails, 92/258 (35.7\%) are positive in culture for the IDFPC when 101/258 (39.2\%) are positive on SAB-ACTI. The difference is not statistically significant $(P$-value $=.27)$. For hair, no difference is observed between the two mediums (6/10, $60 \%$ of positive cultures for both mediums). However among skin samples, the proportion of positive samples is higher for SAB-ACTI medium with $43 / 160$ (26.9\%) of positive samples versus the IDFPC that present $31 / 160$ (19.4\%) of positive samples ( $P$-value $=.0047)$. More precisely regarding dermatophytes, 59/258 (22.9\%) of nails were positive in culture on IDFPC while 79/258 (39.2\%) were positive on SAB-ACTI medium ( $P$ Value $=.0009)$. Among hair samples, there is no difference among tested medium, $6 / 10(60 \%)$ of the samples are positive on both mediums. No statistically significant difference was observed regarding skin samples between both mediums. 26/160 (16.3\%) were positive on IDFPC when $31 / 160$ (19.4\%) were positive on SABACTI for dermatophytes. Regarding contaminants, no statistically 


\begin{tabular}{lccc|} 
& & & $\begin{array}{c}\text { P-value } \\
\text { (McNemar) }\end{array}$ \\
\hline Contaminant & IDFPC & SAB-ACTI & .45 \\
\hline Dermatophyte & $25 / 428(5.8)$ & $30 / 428(7.0)$ & .0002 \\
\hline $\begin{array}{l}\text { Mould potentially pathogen } \\
\text { (direct examination }+ \text { ) }\end{array}$ & $91 / 428(21.3)$ & $116 / 428(27.1)$ & .021 \\
\hline Total & $13 / 428(3.0)$ & $5 / 428(1.2)$ & .024 \\
\hline
\end{tabular}

TABLE 7 Description of the observations of growth observed on both tested media regarding dermatophytes, contaminants and potentially pathogenic moulds

Note: IDFPC:ID-Fungi plates plus cycloheximide, SAB-ACTI-: Sabouraud chloramphenicolcycloheximide medium.

TAB LE 8 Repartition of positive cultures on Id-Fungi plates and Sabouraud depending on the results of direct examination (PAS staining)

\begin{tabular}{|c|c|c|c|}
\hline $\begin{array}{l}\text { Positive cultures in } \\
\text { total }\end{array}$ & IDFPC & SAB-ACTI & $P$-value \\
\hline \multicolumn{4}{|l|}{ (McNemar) } \\
\hline $\begin{array}{l}\text { Direct examination } \\
\text { negative }\end{array}$ & $39 / 279$ (14.0) & $53 / 279$ (19.0) & .048 \\
\hline $\begin{array}{l}\text { Direct examination } \\
\text { positive }\end{array}$ & $90 / 149(60.4)$ & $97 / 149(65.1)$ & .25 \\
\hline \multicolumn{4}{|l|}{$\begin{array}{l}\text { Positive cultures for } \\
\text { dermatophytes }\end{array}$} \\
\hline $\begin{array}{l}\text { Direct examination } \\
\text { negative }\end{array}$ & $14 / 279(5.0)$ & $25 / 279(9.0)$ & .0023 \\
\hline $\begin{array}{l}\text { Direct examination } \\
\text { positive }\end{array}$ & $77 / 149$ (51.7) & $91 / 149$ (61.1) & .013 \\
\hline \multicolumn{4}{|l|}{$\begin{array}{l}\text { Positive cultures } \\
\text { contaminant }\end{array}$} \\
\hline $\begin{array}{l}\text { Direct examination } \\
\text { negative }\end{array}$ & $25 / 279(9.0)$ & $28 / 279$ (10.0) & .64 \\
\hline $\begin{array}{l}\text { Direct examination } \\
\text { positive }\end{array}$ & $0 / 149(0.0)$ & 2/149 (1.3) & .16 \\
\hline \multicolumn{4}{|l|}{$\begin{array}{l}\text { Positive cultures } \\
\text { for potentially } \\
\text { pathogenic moulds }\end{array}$} \\
\hline $\begin{array}{l}\text { Direct examination } \\
\text { negative }\end{array}$ & $0 / 279(0.0)$ & 0/279 (0.0) & \\
\hline $\begin{array}{l}\text { Direct examination } \\
\text { positive }\end{array}$ & 13/149 (8.7) & 4/149 (3.4) & .021 \\
\hline
\end{tabular}

Note: IDFPC:ID-Fungi plates plus cycloheximide, SAB-ACTI: Sabouraud chloramphenicol-cycloheximide medium

significant differences are seen regarding nails and hair comparing both mediums but for skin samples, the rate of contaminants is higher on SAB-ACTI (12/160 7.5\%) than in IDFPC (4/160, 2.5\%) (Pvalue $=.01$ ). Regarding potentially pathogencic moulds from nails, they develop easier on IDFPC $(12 / 258,4.7 \%)$ than on SAB-ACTI $(4 / 258,1.6 \%)(P$-value $=.02)$. Regarding hair and skin samples, they are too few samples to make any conclusion. Table 10 shows the comparison between the concordance between MALDI-TOF MS and ITS sequencing and the concordance between ITS sequencing and microscopy. Between MALDI-TOF and sequencing (129 cases),
TABLE 9 Repartition of positive samples on Id-Fungi plates and Sabouraud medium regarding the type of sample considered (hair, skin, nails)

\begin{tabular}{|c|c|c|c|}
\hline $\begin{array}{l}\text { Positive cultures } \\
\text { in total }\end{array}$ & IDFPC & SAB-ACTI & $P$-value \\
\hline \multicolumn{4}{|l|}{ (McNemar) } \\
\hline Nails & $92 / 258(35.7)$ & $101 / 258(39.2)$ & .28 \\
\hline Hair & $6 / 10(60.0)$ & $6 / 10(60.0)$ & / \\
\hline Skin & $31 / 160(19.4)$ & $43 / 160(26.9)$ & .0047 \\
\hline \multicolumn{4}{|l|}{ Dermatophyte } \\
\hline Nails & $59 / 258(22.9)$ & $79 / 258(30.6)$ & .0009 \\
\hline Hair & $6 / 10(60.0)$ & $6 / 10(60.0)$ & / \\
\hline Skin & $26(16.3)$ & $31 / 160$ (19.4) & .096 \\
\hline \multicolumn{4}{|l|}{ Contaminant } \\
\hline Nails & $21 / 258(8.1)$ & $18 / 258(7.0)$ & .27 \\
\hline Hair & $0 / 10(0.0)$ & $0 / 10(0.0)$ & / \\
\hline Skin & $4 / 160(2.5)$ & $12 / 160(7.5)$ & .011 \\
\hline \multicolumn{4}{|l|}{$\begin{array}{l}\text { Potentially } \\
\text { pathogenic } \\
\text { moulds }\end{array}$} \\
\hline Nails & $12 / 258(4.7)$ & $4 / 258$ (1.6) & .021 \\
\hline Hair & $0 / 10(0.0)$ & $0 / 10(0.0)$ & / \\
\hline Skin & $1 / 160(0.6)$ & $1 / 160(0.6)$ & / \\
\hline
\end{tabular}

Note: IDFPC:ID-Fungi plates plus cycloheximide, SAB-ACTI: Sabouraud chloramphenicol-cycloheximide medium.

15/129 (11.6\%) has discrepant results, 92 (71.4\%) had correct results and 22 are not interpretable (lack of almost one result among both methods). Regarding the comparison between microscopy and ITS sequencing, 19 (19.8\%) results were discrepant, 70 (72.9\%) were concordant between both methods and 7 (7.3\%) were not interpretable. No statistically significant difference was seen among both methods for discrepant results.

\subsection{1 | MALDI-TOF MS results}

All the samples that were positive on IDFPC were identified in parallel by MALDI-TOF MS (using Bruker 2.0 filamentous fungi database) and ITS sequencing or DermaGenius. Samples that were positive on 
TABLE 10 Comparison of the concordances of results obtained with respect to ITS sequencing of culture on Id-Fungi plates followed by MALDI-TOF MS and on culture on SAB-ACTI medium followed by microscopy

\begin{tabular}{|lllll|}
\hline Identifications & $\begin{array}{l}\text { Discrepant } \\
\text { results (\%) }\end{array}$ & $\begin{array}{l}\text { Concordant } \\
\text { results (\%) }\end{array}$ & $\begin{array}{l}\text { Not interpretable } \\
\text { (\%) }\end{array}$ & Total (\%) \\
\hline $\begin{array}{l}\text { MALDI-TOF MS vs } \\
\text { sequencing }\end{array}$ & $15(11.6)$ & $92(71)$ & $22(17.1)$ & $129(100.0)$ \\
\hline \begin{tabular}{l} 
Sequencing vs microscopy \\
\hline
\end{tabular} & $19(19.8)$ & $70(72.9)$ & $7(7.3)$ & $96(100.0)$ \\
\hline
\end{tabular}

FIGURE 1 MALDI-TOF MS identification rate among the 129 positive cultures on ID-Fungi plates plus cycloheximide (IDFPC)
FIGURE 2 Distribution of principal MALDI-TOF identifications among the positive cultures of dermatophytes on IDFPC
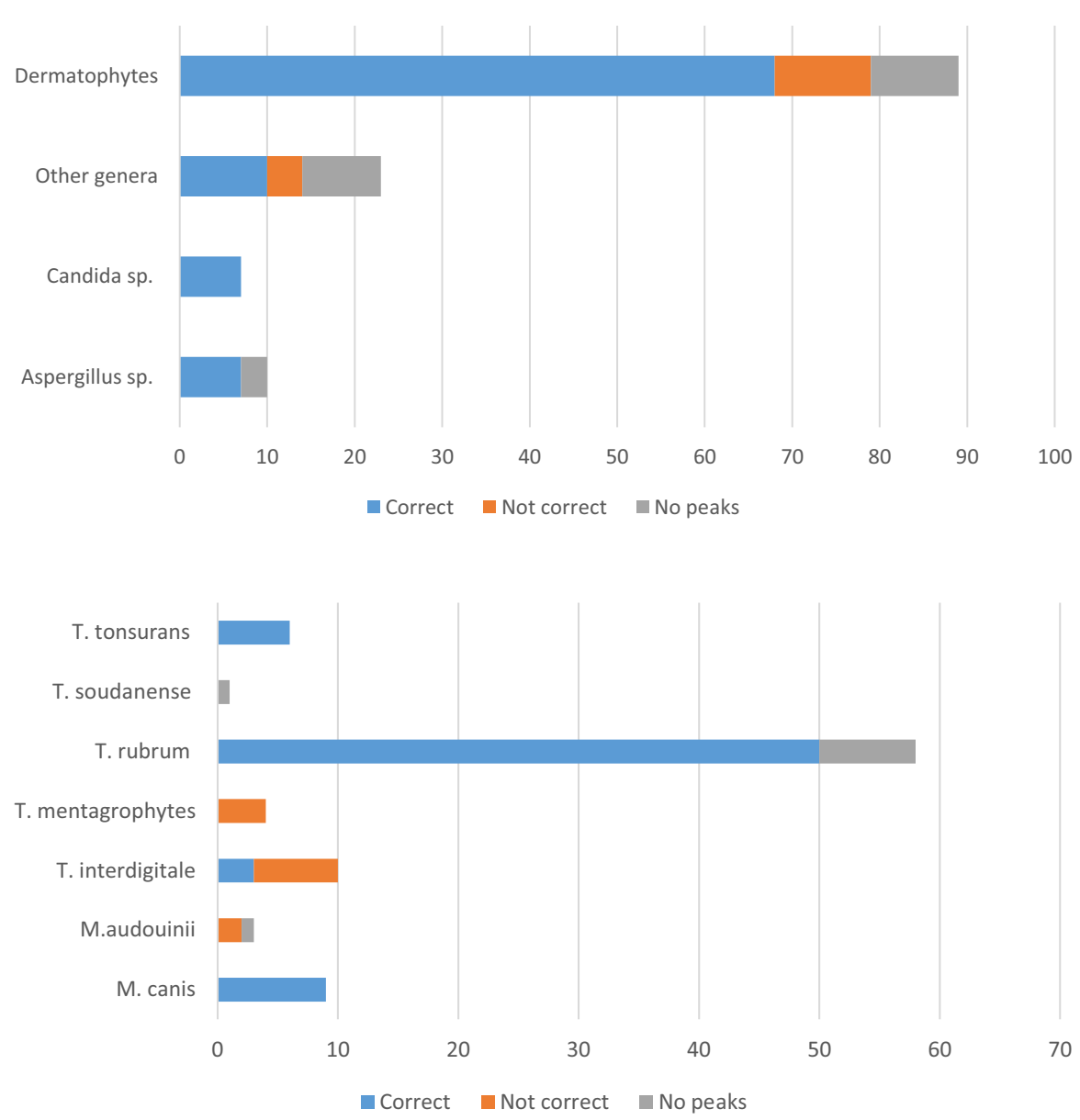

SAB-ACTI medium were identified by microscopy and in some cases by ITS sequencing or DermaGenius in the same way as it is done in our routine laboratory. Results are presented in Figure 1. Among the 129 positive cultures on IDFPC, 92 (71.4\%) showed a correct identification by MALDI-TOF MS confirmed by ITS PCR or DermaGenius while 15 (11.6\%) showed an incorrect identification, and 22 (17.1\%) had no peaks.

Regarding dermatophytes (see Figure 2), 68/91 strains (75.2\%) were correctly identified. If we exclude strains that are not present on the Bruker 2.0 database, 68/83 (83.3\%) were correctly identified by MALDI-TOF MS. Among T rubrum strains defined by ITS sequencing or DermaGenius, 50/58 (86\%) were correctly identified by MALDI-TOF MS, 8/58 (14\%) did not give any peaks. Even with complete ethanol-formic acid extraction, no peak was obtained for these 8 strains. Regarding $T$ interdigitale, 10 strains were identified with ITS sequencing or DermaGenius. Among these, 3/10 (30\%) obtained a correct identification by MALDI-TOF MS. The other 7 were incorrectly identified as $T$ tonsurans (70\%). Four strains of $T$ mentagrophytes were characterised on this study, but none were correctly identified by MALDI-TOF MS. They were incorrectly identified as $T$ tonsurans (1), $T$ interdigitale (2) or $T$ equinum (1). Trichophyton mentagrophytes species is not present on Bruker 2.0 database. The 6 strains of $T$ tonsurans were all correctly identified (100\%). 9/9 strains of $M$ canis were correctly identified by MALDI-TOF MS (100\%). Three strains of $M$ audouinii were identified by ITS sequencing or DermaGenius. Two of them were incorrectly identified as $M$ canis, and the other one gave no peaks by MALDI-TOF MS This species is not present on Bruker 2.0 filamentous fungi database as $T$ soudanense. The only strain of $T$ soudanense was not identified (no peaks). Some Aspergillus were identified on Id-Fungi plates. 7/10 (70\%) showed a correct identification by MALDI-TOF MS. More precisely, 4/4 strains of $A$ fumigatus were correctly identified by MALDI-TOF MS, $1 / 1$ strain of $A$ flavus was correctly identified, $1 / 2$ strains of $A$ terreus and $A$ versicolor presented a correct identification. The other 
one had no peaks. The unique strain of $A$ sidowii was not identified (no peaks).

Regarding yeasts, 7 strains grown on Id-Fungi plates. 7/7(100\%) gave correct identifications by MALDI-TOF MS. 4/4 of Candida albicans strains were correctly identified. $2 / 2$ strains of Candida parapsilosis and 1 strain of Candida guillermondii were well identified.

Other species were identified sporadically and are not described in details here. Among these, a lot of species that gave 'no peaks' were not on the MALDI-TOF MS database.

\section{4 | DISCUSSION}

In this study, we have evaluated the performance of a new commercialised media that can help for the identification of filamentous fungi by MALDI-TOF MS. Indeed, if bacteria and yeasts are currently easily and accurately identified by MALDI-TOF MS, for filamentous fungi, its wider application has been very limited. The most frequent limitation is the availability of complete databases including filamentous fungi species and a lack of standardised procedures for fungal analysis. The IDFP medium evaluated in this study can be a tool to help for standardisation because of a low adherence membrane facilitating the colony harvest. Indeed in the literature, a lot of different schemes have been used for filamentous fungi identification. For Aspergillus, non-Aspergillus moulds and dermatophytes, several different protocols have already been described using liquid or solid media, different times of incubation and different methods of extraction. ${ }^{7,8,15-21}$ Liquid media has sometimes been described to be better than solid media as it permits to obtain a more homogeneous mycelium, easier to be emulsified and rendering better quality spectra. ${ }^{15,22,23}$ This study shows that the use of a solid media can also give rise to good rates of identification. Indeed among the Aspergillus species, $92 \%$ of correct identifications were obtained. For these genera, a complete formic acid acetonitrile extraction gives rise to better results after inoculation on the IDFP medium and 48-hour incubation. The rate of correct identification obtained for Aspergillus with IDFP medium can be considered as good regarding rates already described in the literature that are very variable ranging from $0 \%$ to $100 \% \cdot{ }^{16,19,22,24-26}$ For non-Aspergillus species, a rate of $73 \%$ of correct identification was obtained after 24 hours following complete formic acid acetonitrile extraction. So also in this case, protein extraction is encouraged to obtain optimal results. Regarding dermatophytes, surprisingly, the best rates of correct identification were obtained with an extended direct deposit after 72 hours of incubation where $78 \%$ of correct identification was obtained with the IDFP medium. This is a new fact according to publications on dermatophyte identification by MALDI-TOF MS where protein extraction gives in general better results than extended direct deposit. ${ }^{6-8,21,27}$ However, two studies recently assessed the ability to identify fungi by MALDI-TOF MS using a direct deposit method. ${ }^{28,29}$ The rate of $78 \%$ of correct identification obtained for dermatophytes using the IDFP medium is satisfactory. L'Ollivier et al described in one review ten studies published between 2008 and 2015 showing accuracy of identification by MALDI-TOF MS ranging from $14 \%$ to $100 \%$ for dermatophytes. ${ }^{7}$ Rates more than $90 \%$ for dermatophytes are nevertheless obtained under different conditions in some studies. ${ }^{20,30,31}$ However, most studies give good rates of identification after several days of culture sometimes reaching 30 days. So obtaining $78 \%$ of identification after 3 days of culture is noteworthy. The comparison of the IDFP medium with SGC2 solid medium shows that for Aspergillus, non-Aspergillus moulds and dermatophytes, the rates of correct identification obtained following diverse tested conditions (extraction/extended direct deposit and different times tested) were clearly better by using IDFP medium than by using SGC2. We also confirmed the quicker manipulation, announced by the firm Conidia for the deposit with this new medium as the culture is easily taken with the IDFP medium almost as easy as for bacteria or yeasts thanks to the presence of the membrane that avoid adherence of the culture. This is time saving compared to SGC2 that imply the use of a scalpel to snatch the culture with the risk to take agar pieces that can give rise to bad MALDI-TOF MS identification.

Some studies described that decreasing the species cut-off to 1.7 increased species identification level without leading to misidentification. ${ }^{13,32,33}$ We can observe the same fact in our study so the cut-off value for results was set at 1.7, and we encourage other laboratories to use the same cut-off to improve correct fungal identification by MALDI-TOF MS.

A lot of studies pointed out the need for adapted databases and the need to expand the Bruker current filamentous fungi database with new species and a greater number of entries to improve the sensitivity of the MALDI-TOF MS identification for dermatophytes $7,8,20,30,34,35$ but also for the other filamentous fungi. ${ }^{13,16,17,24,36}$ For this reason, we evaluated the results obtained by using the IDFP medium with the web-based MSI application comparing the identification results with those obtained by only using the original Bruker filamentous fungi 2.0 database. MSI database has been previously evaluated to improve correct identification of filamentous fungi, including dermatophytes. ${ }^{14,37}$ In our hands, the use of IDFP medium combined with the MSI database did not significantly improve the results obtained for dermatophytes identification. Indeed, the higher rate of correct identification (78\%) obtained with the Bruker 2.0 filamentous fungi database is not improved in any conditions tested, by using MSI database. Using Bruker database, we clearly showed a lack of spectra for some species such as $M$ audouinii or $T$ soudanense that are the two most represented dermatophytes giving rise to tinea capitis in Belgium but also in other European countries. ${ }^{38-43}$ Trichophyton violaceum another agent of tinea capitis is present with only one strain in the Bruker database. Trichophyton mentagrophytes one zoophilic strain, recognised to be different than $T$ interdigitale following the new classification of the Hoog et al, ${ }^{44}$ is not represented either. This strain is acquiring terbinafine resistance giving epidemics in India, and it is particularly of interest to distinguish this strain from the anthropophilic one $T$ interdigitale. ${ }^{45,46}$ All these species are well present in the MSI database. However, as these species were not present on the Bruker filamentous fungi 
database, they have been excluded from the present study, but among the tested species, there was no clear improvement of dermatophytes identification using MSI application. The present study showed some weaknesses of the Bruker database as T interdigitale is cross-reacting with $T$ tonsurans. Indeed, some strains of $T$ interdigitale are recognised as $T$ tonsurans with scores above 2 . This can clearly lead to misinterpretation of results by novice laboratories, or for laboratories with no training in conventional mycological methods such as microscopy. This cross-reaction is not improved by using the MSI database as other species are cross-reacting with $T$ interdigitale such as $T$ batonrougeii, $T$ mentagrophytes and $T$ tonsurans. The same problem is seen for $T$ tonsurans strains being submitted to the MSI database. However, Bruker database seems to better recognise this species avoiding cross-reactions for these $T$ tonsurans strains. The cross-reaction described is probably due to the high similarity in protein expression between these closely related species belonging to the same " clade» defined by the Hoog et $\mathrm{al}^{44}$ Major peaks present in $T$ tonsurans mass spectra are also present in $T$ interdigitale. Cross-reactions between abovecited species have already been discussed in the literature. ${ }^{6,7}$ The fact that MSI application contains more species spectra can give rise to increased cross-reactions as other species closed to $T$ interdigitale such as $T$ batonrougeii or T mentagrophytes are also present in the database, increasing confusion possibilities. One strain of $T$ violaceum included in this study was misidentified as $T$ rubrum by MSI database. However, both evaluated databases could well distinguish among $T$ interdigitale and $T$ benhamiae that, according to the re-evaluated taxonomy of dermatophytes, are differentiated into two different clades. ${ }^{44}$ So this study shows that while several efforts have been done to improve databases for dermatophytes, results given by the MALDI-TOF MS should be taken with precaution and a total skip of the culture methods is not recommended for laboratories that need a precise diagnostic of dermatophytes infection.

Regarding Aspergillus species, good results were obtained using Bruker database (12/13 of correct ID), and similar results were obtained by MSI use (12/13). However, for non-Aspergillus species, the use of MSI database after 48 hours of incubation significantly increases the rate of correct identification to $92 \%$ compared to $73 \%$ under the best conditions with Bruker 2.0 Filamentous fungi database. This can be explained with the wide range of filamentous fungi spectra present on this web-based application, and some species such as Acremonium sp and Rasamsonia sp were few represented giving rise to no/bad identification with Bruker database. So regarding this study, MSI database use is encouraged for the identification of Aspergillus and non-Aspergillus moulds as it can improve (mostly for non-Aspergillus moulds), the rate of correct identifications obtained by using the IDFP medium compared to Bruker filamentous fungi 2.0 database.

It has been shown in the literature that the way by which initial spectra are acquired is very important as growth conditions greatly influence mass spectra, ${ }^{47-49}$ so one way to increase performances of the IDFP medium should be to create a homemade database based on the same culture features. Indeed, Bruker database is created using liquid cultures while MSI is based on solid agar medium cultures. This can explain the higher rates of correct identifications obtained under certain conditions with MSI medium.

Regarding the culture of nails hair and skin performed directly on IDFPC, our results demonstrate that this tool permits to obtain globally $71.4 \%$ of correct identification by MALDI-TOF MS and $75 \%$ of dermatophytes are well identified in a short time and using an extended direct deposit. The average for positivity on this medium (and so to obtain an identification by MALDI-TOF MS) was 6 days. This is a positive point regarding conventional methods that are time-consuming, with a turnaround time that can be up to 3 weeks and need for huge technician training and expertise for microscopic identification. However, we have seen some weaknesses of the IDFPC medium compared to SAB-ACTI medium. Indeed, dermatophytes develop more easily on SAB-ACTI than on IDFPC. Our results indeed show a statistically significant difference on the rate of positive cultures for dermatophytes between both media. Moreover, among samples that showed a negative direct examination, a higher rate was then positive in culture on SAB-ACTI than in IDFPC. On the contrary, non dermatophytes moulds pottentially pathogenic (samples with a positive direct examination) develop easier on IDFPC than on SAB-ACTI. Regarding contaminations (negative direct examination with a positive non-dermatophyte culture), the rate is similar on both mediums.

A lack of adherence of nails on the IDFPC medium has been observed, giving rise to sometimes loss of material or growth outside the membrane, what is problematic for MALDI-TOF MS deposit. Furthermore, the plates are drying much faster than the slant agar tubes. This is also a problem regarding the fact that some dermatophytes, in particular those from hair samples, need several weeks to develop as M audouinii or T soudanense. The sporulation on IDFPC has also been evaluated to be lower than on SAB-ACTI medium making difficult the completion of the identification given by MALDITOF MS with microscopy. Regarding results of the identifications of dermatophytes obtained by culture on IDFPC, the rates obtained are satisfactory (75\%). $100 \%$ of correct identification were obtained for $M$ canis and $T$ tonsurans. A rate of $86 \%$ of correct identification was observed for $T$ rubrum. 14\% of $T$ rubrum showed no peaks but no misidentification was observed. The identification of $T$ interdigitale remains problematic as discussed above. Following our observations, we think that the composition of the medium should be changed to be closer to SAB-ACTI medium tested in parallel in this study. Or maybe the composition of the medium is not a problem but the presence of the membrane decreases the sensitivity of the medium. Moreover, the sporulation of the medium should be improved to permit in parallel MALDI-TOF MS identification and identification by microscopy. The adherence of nails on the membrane should also be improved. The combination of these should be optimal for fast and accurate dermatophyte identification.

One great advantage for the two versions of Id-Fungi plates medium tested is that the presence of a membrane present at the surface of the culture medium prevents the adherence of fungi 
and allows an easier colony harvest. This is a good thing that can be applied to facilitate deposit on MALDI-TOF MS target but also for other application such as DNA extraction avoiding contaminations. Indeed, liquid culture medium is often used for DNA extraction of fungi, giving rise to better rates of DNA amounts. However, liquid culture medium is a source of contaminations post-inoculation by fungi present in the environment. Using a media such Id-Fungi plates medium may avoid these contaminations, giving rise to an easy take of the fungal culture based on solid media. Using IDFPC as the only culture medium is not recommended as the sensitivity of this medium seems lower than with the Sabouraud medium usually used in our laboratory. However this medium can be useful as a supplementary tool for the rapid identification of dermatophytes. In case of doubts with microscopy, it can help for the final decision.

\section{5 | CONCLUSION}

According to the results obtained in the present study, we can assess the utility of IDFP medium to improve rates of correct identification obtained for filamentous fungi with the MALDI-TOF MS. The medium allows an easy deposit on the MALDI-TOF MS plate and gives significantly better rates of identification than that also evaluated SGC2 medium. IDFP medium allows to obtain satisfactory rates of identification in less than 72 hours for Aspergillus, non-Aspergillus moulds and dermatophytes. This is remarkable considering conventional methods where we can wait for more than 3 weeks to obtain some reliable results for slow-growing moulds. The use of an enriched database such as MSI application seems to be useful for Aspergillus and non-Aspergillus moulds but still need to be improved concerning dermatophytes following our observations. The refinement of the performance of the IDFPC medium seems necessary to improve the sensitivity of this medium and permit to consider a direct culture of nails, hair and skin samples preceding an identification by MALDI-TOF MS.

\section{CONFLICT OF INTEREST}

The authors declare no conflicts of interest.

\section{AUTHOR CONTRIBUTIONS}

Rosalie Sacheli: Conceptualization (equal); data curation (supporting); formal analysis (lead); investigation (equal); methodology (equal); supervision (equal); validation (equal); visualization (equal); writing-original draft (lead). Anne-Sophie Henri: Investigation (equal), Methodology (equal). Laurence Seidel: Data curation (equal); validation (equal). Marie Ernst: Data curation (equal); validation (equal). Rajae Darfouf: Investigation (equal), methodology (equal). Akole Adjetey Bahun: Investigation (equal), methodology (equal). Marjorie Schyns: Investigation (equal), methodology (equal). Ludwig Marechal: Investigation (equal), methodology (equal). Jorge Arrese: Investigation (equal); validation (supporting). Cécile Meex: Conceptualization (equal); methodology (equal); validation (equal), writing original draft (supporting). Marie-Pierre Hayette:
Conceptualization (equal); project administration (equal); supervision (equal); validation (equal); writing-original draft (supporting).

\section{DATA AVAILABILITY STATEMENT}

None.

\section{ORCID}

Rosalie Sacheli (iD https://orcid.org/0000-0002-3922-324X

\section{REFERENCES}

1. Carbonnelle E, Mesquita C, Bille E, et al. MALDI-TOF mass spectrometry tools for bacterial identification in clinical microbiology laboratory. Clin Biochem. 2011;44:104-109.

2. Bizzini A, Greub G. Matrix-assisted laser desorption ionization time-of-flight mass spectrometry, a revolution in clinical microbial identification. Clin Microbiol Infect. 2010;16:1614-1619.

3. Clark AE, Kaleta EJ, Arora A, Wolk DM. Matrix-Assisted laser desorption ionization-time of flight mass spectrometry: a fundamental shift in the routine practice of clinical microbiology. Clin Microbiol Rev. 2013;26:547-603.

4. Dhiman N, Hall L, Wohlfiel SL, Buckwalter SP, Wengenack NL. Performance and cost analysis of matrix-assisted laser desorption ionization-time of flight mass spectrometry for routine identification of yeast. J Clin Microbiol. 2011;49:1614-1616.

5. Van Veen SQ, Claas ECJ, Kuijper EJ. High-throughput identification of bacteria and yeast by matrix-assisted laser desorption ionization-time of flight mass spectrometry in conventional medical microbiology laboratories. J Clin Microbiol. 2010;48:900-907.

6. Hedayati MT, Ansari S, Ahmadi B, et al. Identification of clinical dermatophyte isolates obtained from Iran by matrix-assisted laser desorption/ionization time-of light mass spectrometry. Curr Med Mycol. 2019;5(2):22-26

7. L'Ollivier C, Ranque S. MALDI-TOF-Based Dermatophyte Identification. Mycopathologia. 2017;182(1-2):183-192.

8. Nenoff P, Erhard M, Simon JC, et al. MALDI-TOF mass spectrometry - a rapid method for the identification of dermatophyte species. Med Mycol. 2013;51:17-24.

9. De Respinis S, Monnin V, Girard V, et al. Matrix-assisted laser desorption ionization-time of flight (MALDITOF) mass spectrometry using the Vitek MS system for rapid and accurate identification of dermatophytes on solid cultures. J Clin Microbiol. 2014;52:4286-4292.

10. Lau AF, Drake SK, Calhoun LB, Henderson CM, Zelazny AM. Development of a clinically comprehensive database and a simple procedure for identification of molds from solid media by matrix-assisted laser desorption ionization-Time of flight mass spectrometry. J Clin Microbiol. 2013;51:828-834.

11. Lachaud L, Fernández-Arévalo A, Normand AC, et al. Identification of Leishmania by MALDI-TOF mass spectrometry using a free web-based application and a dedicated mass spectral library. J Clin Microbiol. 2017;55(10):2924-2933.

12. Ferrer C, Colom F, Frasés S, Mulet E, Abad JL, Alió JL. Detection and identification of fungal pathogens by PCR and by ITS2 and 5.8S ribosomal DNA typing in ocular infections. J Clin Microbiol. 2001;39:2873-2879.

13. Normand A-C, Cassagne C, Gautier M, et al. Decision criteria for MALDI-TOF MS-based identification of filamentous fungi using commercial and in-house reference databases. BMC Microbiol. 2017;17(1):25.

14. Normand AC, Becker P, Gabriel F, et al. Validation of a new web application for identification of fungi by use of matrix-assisted laser desorption ionization-time of flight mass spectrometry. J Clin Microbiol. 2017;55:2661-2670. 
15. Vidal-Acuña MR, Ruiz-Pérez De Pipaón M, Torres-Sánchez MJ, Aznar J. Identification of clinical isolates of Aspergillus, including cryptic species, by matrix assisted laser desorption ionization time-of-flight mass spectrometry (MALDI-TOF MS). Med Mycol. 2018;56:838-846.

16. De Carolis E, Posteraro B, Lass-Flörl C, et al. Species identification of Aspergillus, Fusarium and Mucorales with direct surface analysis by matrix-assisted laser desorption ionization time-of-flight mass spectrometry. Clin Microbiol Infect. 2012;18:475-484.

17. Park JH, Shin JH, Choi MJ, et al. Evaluation of matrix-assisted laser desorption/ionization time-of-fight mass spectrometry for identification of 345 clinical isolates of Aspergillus species from 11 Korean hospitals: comparison with molecular identification. Diagn Microbiol Infect Dis. 2017;87:28-31.

18. McMullen A, Wallace M, Pincus D, Wilkey K, Burnham C-A. Evaluation of the VITEK MS MALDI-TOF MS system for identification of clinically relevant filamentous fungi. J Clin Microbiol. 2016;54(8):2068-2073.

19. Rychert J, Slechta ES, Barker AP, et al. Multicenter evaluation of the Vitek MS v3.0 system for the identification of filamentous fungi. $J$ Clin Microbiol. 2018;56(2):e01353-17

20. Packeu A, De Bel A, I'Ollivier C, Ranque S, Detandt M, Hendrickx M. Fast and accurate identification of dermatophytes by MALDI-TOF mass spectrometry: validation in the clinical lab. J Clin Microbiol. 2014;52(12):4286-4292.

21. Intra J, Sarto C, Tiberti N, Besana S, Savarino C, Brambilla P. Genuslevel identification of dermatophytes by MALDI-TOF MS after 2 days of colony growth. Lett Appl Microbiol. 2018;67:136-143.

22. Schulthess B, Ledermann R, Mouttet F, et al. Use of the Bruker MALDI Biotyper for identification of molds in the clinical mycology laboratory. J Clin Microbiol. 2014;52:2797-2803.

23. Reich M. Species identification of bacteria and fungi from solid and liquid culture media by MALDI-TOF mass spectrometry. $J$ Bacteriol Parasitol. 2013;01:S5-002.

24. Masih A, Singh PK, Kathuria S, Agarwal K, Meis JF, Chowdhary A. Identification by molecular methods and matrix-assisted laser desorption ionization-time of flight mass spectrometry and antifungal susceptibility profiles of clinically significant rare aspergillus species in a referral chest hospital in Delhi, India. J Clin Microbiol. 2016;54:2354-2364.

25. Stein M, Tran V, Nichol KA, et al. Evaluation of three MALDI-TOF mass spectrometry libraries for the identification of filamentous fungi in three clinical microbiology laboratories in Manitoba, Canada. Mycoses. 2018;61:743-753.

26. Patel R. A moldy application of MALDI: MALDI-ToF mass spectrometry for fungal identification. J Fungi. 2019;5:4.

27. L'Ollivier C, Cassagne C, Normand A-C, et al. A MALDI-TOF MS procedure for clinical dermatophyte species identification in the routine laboratory. Med Mycol. 2013;51:713-720.

28. Zvezdanova ME, Escribano P, Ruiz A, et al. Increased species-assignment of filamentous fungi using MALDI-TOF MS coupled with a simplified sample processing and an in-house library. Med Mycol. 2019;57:63-70.

29. da Cunha KC, Riat A, Normand A-C, et al. Fast identification of dermatophytes by MALDI-TOF/MS using direct transfer of fungal cells on ground steel target plates. Mycoses. 2018;61:691-697.

30. Calderaro A, Motta F, Montecchini S, et al. Identification of Dermatophyte Species after implementation of the in-house MALDI-TOF MS database. Int J Mol Sci. 2014;15:16012-16024.

31. Karabıçak N, Karatuna O, İlkit M, Akyar I. Evaluation of the bruker matrix-assisted laser desorption-ionization time-of-flight mass spectrometry (MALDI-TOF MS) system for the identification of clinically important dermatophyte species. Mycopathologia. 2015;180:165-171.

32. Sleiman S, Halliday CL, Chapman B, et al. Performance of matrix-assisted laser desorption ionization time of flight mass spectrometry for identification of aspergillus, scedosporium, and fusarium spp. in the australian clinical setting. J Clin Microbiol. 2016;54:2182-2186.

33. Becker P, Normand A-C, Vanantwerpen G, et al. Identification of fungal isolates by MALDI-TOF mass spectrometry in veterinary practice: validation of a web application. J Vet Diagnostic Investig. 2019;31:471-474.

34. De Respinis S, Tonolla M, Pranghofer S, Petrini L, Petrini O, Bosshard PP. Identification of dermatophytes by matrix-assisted laser desorption/ionization time-of-flight mass spectrometry. Med Mycol. 2013;51:514-521.

35. De Respinis S, Monnin V, Girard V, et al. MALDI-TOF VITEK MS for the rapid and accurate identification of dermatophytes on solid cultures. J Clin Microbiol. 2014;52(12):4286-4292.

36. Lau AF, Walchak RC, Miller HB, et al. Multicenter study demonstrates standardization requirements for mold identification by MALDI-TOF MS. Front Microbiol. 2019;10:2098.

37. Cassagne C, Normand AC, L'Ollivier C, Ranque S, Piarroux R. Performance of MALDI-TOF MS platforms for fungal identification. Mycoses. 2016;59:678-690.

38. Vanbeckhoven D., Sacheli R., Patteet S., Hayette MP, Lagrou K. 2016-2017 report of the Belgian National Reference Center for Mycoses. 2019 https://doi.org/10.25608/0eb9-2439

39. Sacheli R, Adjetey C, DarfoufR, et al. A one-year survey of Microsporum audouinii infections in Belgium: epidemiological and genotypic characterization. Clin Microbiol Infect. 2016;22:285.e9-285.e17.

40. Brito-Santos F, Figueiredo-Carvalho MHG, Coelho RA, Sales A, Almeida-Paes R. Tinea Capitis by Microsporum audouinii: case reports and review of published global literature 2000-2016. Mycopathologia. 2017;182:1053-1060.

41. Ginter-Hanselmayer G, Weger W, Ilkit M, Smolle J. Epidemiology of tinea capitis in Europe: current state and changing patterns. Mycoses. 2007;50:6-13.

42. Panasiti V, Devirgiliis V, Borroni RG, et al. Epidemiology of dermatophytic infections in Rome, Italy: a retrospective study from 2002 to 2004. Med Mycol. 2002;45:57-60.

43. Rezusta A, Betrán A, Querol I, Palacián MP, Revillo MJ. Tinea capitis caused by Trichophyton soudanense and Microsporum audouinii in an adult: a case report. Mycoses. 2011;54:89-90.

44. de Hoog GS, Dukik K, Monod M, et al. Toward a novel multilocus phylogenetic taxonomy for the Dermatophytes. Mycopathologia. 2017;182:5-31.

45. Yamada T, Maeda M, Alshahni MM, et al. Terbinafine resistance of Trichophyton clinical isolates caused by specific point mutations in the squalene epoxidase gene. Antimicrob Agents Chemother. 2017;61(7):e00115-17.

46. Singh A, Masih A, Khurana A, et al. High terbinafine resistance in Trichophyton interdigitale isolates in Delhi, India harbouring mutations in the squalene epoxidase gene. Mycoses. 2018;61(7):477-484.

47. Cassagne C, Ranque S, Normand A-C, et al. Mould routine identification in the clinical laboratory by Matrix-Assisted laser desorption ionization Time-Of-Flight mass spectrometry. PLoS One. 2011;6(12):e28425.

48. Posteraro B, De Carolis E, Vella A, Sanguinetti M. MALDI-TOF mass spectrometry in the clinical mycology laboratory: Identification of fungi and beyond. Expert Rev Proteomics. 2013;10(2):151-164.

49. Bader O. MALDI-TOF-MS-based species identification and typing approaches in medical mycology. Proteomics. 2013;13:788-799.

How to cite this article: Sacheli R, Henri A-S, Seidel L, et al. Evaluation of the new Id-Fungi plates from Conidia for MALDI-TOF MS identification of filamentous fungi and comparison with conventional methods as identification tool for dermatophytes from nails, hair and skin samples. Mycoses. 2020;00:1-13. https://doi.org/10.1111/myc.13156 